\title{
Effects of dietary calcium to phosphorus ratio and addition of phytase on growth performance of nursery pigs ${ }^{1}$
}

\author{
Fangzhou Wu, ${ }^{*, 2}$ Mike D. Tokach, ${ }^{*}$ Steve S. Dritz, ${ }^{\dagger}$ Jason C. Woodworth, ${ }^{*}$ Joel M. DeRouchey, \\ Robert D. Goodband, ${ }^{*}$ Marcio A. D. Gonçalves, ${ }^{\ddagger}$ and Jon R. Bergstrom ${ }^{\S}$
}

* Department of Animal Sciences and Industry, College of Agriculture, Kansas State University, Manhattan 66506; 'Department of Diagnostic Medicine/Pathobiology, College of Veterinary Medicine, Kansas State University, Manhattan 66506; ${ }^{\ddagger}$ Genus PIC, Hendersonville, TN 37075; ${ }^{\circledR}$ DSM Nutritional Products Inc., Parsippany, NJ 07054

\begin{abstract}
Two studies were conducted to evaluate the growth performance and percentage bone ash of nursery pigs fed various combinations of $\mathrm{Ca}$ and $\mathrm{P}$ provided by inorganic sources or phytase. In Exp. 1, pens of pigs ( $n=720$, initially $6.1 \pm 0.98 \mathrm{~kg})$ were blocked by initial BW. Within blocks, pens were randomly assigned to one of six treatments (12 pens per treatment) in a three-phase diet regimen. Treatments were arranged in a $2 \times 3$ factorial with main effects of $\mathrm{Ca}(0.58 \%$ vs. $1.03 \%)$ and standardized total tract digestible (STTD) P $(0.33 \%$ and $0.45 \%$ without phytase, and $0.45 \%$ with $0.12 \%$ of the $\mathrm{P}$ released by phytase). During treatment period, $\mathrm{Ca}$ $\times$ P interactions were observed for all growth criteria $(P<0.05)$. When diets had low $\mathrm{Ca}$, pigs fed $0.45 \%$ STTD $P$ with phytase had greater $(P<0.01)$ ADG and ADFI than those fed $0.33 \%$ or $0.45 \%$ STTD P without phytase. When high Ca was fed, ADG and ADFI were similar among pigs fed $0.45 \%$ STTD $\mathrm{P}$ with or without phytase and were greater than those fed $0.33 \%$ STTD P. Gain:feed was reduced $(P<0.01)$ when high $\mathrm{Ca}$ and low STTD $\mathrm{P}$ were fed relative to other treatments. On d 21, radiuses were collected from 1 pig per pen for bone ash analysis. Pigs fed $0.33 \%$ STTD P had decreased $(P<0.05)$ percentage bone ash than those fed $0.45 \%$ STTD $P$ with or without phytase when high $\mathrm{Ca}$ was fed, but

this $\mathrm{P}$ effect was not observed for low $\mathrm{Ca}$ diets $(\mathrm{Ca}$ $\times \mathrm{P}$ interaction, $P=0.007)$. In Exp. 2,36 pens $(10$ pigs per pen, initially $6.0 \pm 1.08 \mathrm{~kg}$ ) were used in a completely randomized design. Treatments were arranged in a $2 \times 3$ factorial with the main effects of STTD P (at or above NRC [NRC. 2012. Nutrient Requirements of Swine. $11^{\text {th }}$ rev. ed. Washington (DC): National Academic Press.] requirement estimates) and total $\mathrm{Ca}(0.65,0.90$, and $1.20 \%)$. Experimental diets were fed during phases 1 and 2, followed by a common phase 3 diet. Diets at NRC (2012) P level contained $0.45 \%$ and $0.40 \%$ STTD P, compared with $0.56 \%$ and $0.52 \%$ for diets greater than the NRC (2012) estimates, in phase 1 and 2, respectively. During treatment period, increasing Ca decreased (linear, $P=0.006$ ) ADG, but increasing STTD $P$ marginally increased $(P=0.084)$ ADG, with no $\mathrm{Ca} \times \mathrm{P}$ interaction. When diets contained NRC (2012) P levels, pigs fed $1.20 \% \mathrm{Ca}$ had decreased $(P<0.05) \mathrm{G}: \mathrm{F}$ than those fed $0.65 \%$ or $0.90 \% \mathrm{Ca}$; however, when high STTD P were fed, $\mathrm{G}: \mathrm{F}$ was not affected by $\mathrm{Ca}(\mathrm{Ca} \times \mathrm{P}$ interaction, $P=0.018)$. In conclusion, excess $\mathrm{Ca}$ decreased pig growth and percentage bone ash when diets were at or below NRC (2012) requirement for STTD P, but these negative effects were alleviated by adding monocalcium $\mathrm{P}$ or phytase to the diet.
\end{abstract}

Key words: bone ash, calcium, growth performance, nursery pig, phosphorus, phytase

(C) The Author (s) 2018. Published by Oxford University Press on behalf of the American Society of Animal Science. All rights reserved. For permissions, please e-mail: journals.permissions@oup.com.

J. Anim. Sci. 2018.96:1825-1837 doi: 10.1093/jas/sky101

\footnotetext{
${ }^{1}$ Appreciation is expressed to DSM Nutritional Products, Inc. (Parsippany, NJ) and PIC North America (Hendersonville, TN) for their technical support and partial funding. Special appreciation is also expressed to Julie Salyer, Dr Brad James, and Lorene Parkhurst from Kalmbach Feeds,
}

Inc. (Sycamore, $\mathrm{OH})$, for their technical support and expertise in conducting the experiment.

${ }^{2}$ Corresponding author: fangzhou@ksu.edu

Received January 22, 2018.

Accepted March 16, 2018. 


\section{INTRODUCTION}

Appropriate dietary $\mathrm{Ca}$ and $\mathrm{P}$ concentrations are essential for nursery pig performance. Accurate formulation for $\mathrm{Ca}$ and $\mathrm{P}$ is even more important in recent years with the routine use of phytase in swine diets. Research has demonstrated that feeding excess dietary $\mathrm{Ca}$ impairs $\mathrm{P}$ absorption, resulting in reduced growth performance and bone calcification of pigs (Reinhart and Mahan, 1986; Stein et al., 2011; González-Vega et al., 2016). This effect is especially prominent when diets are marginal in P (Létourneau-Montminy et al., 2012; NRC, 2012). Moreover, excess $\mathrm{Ca}$ can bind to phytate in the small intestine, decrease the solubility of phytate, and therefore impair the ability of phytase to release $\mathrm{P}$ (Dersjant-Li et al., 2015).

Diets can have excess $\mathrm{Ca}$ for multiple reasons, including formulation errors, variability in laboratory analysis of ingredients, neglecting the $\mathrm{Ca}$ content of carriers in premixes or other additives, and not accounting for $\mathrm{Ca}$ released by phytase. Meanwhile, diets can also be deficient in $\mathrm{P}$ due to formulation errors or by overestimating the amount of $\mathrm{P}$ released for the given amount of phytase in the diet. In addition, recent research (Vier et al., 2017) has suggested that NRC (2012) may underestimate the standardized total tract digestible (STTD) P requirement for nursery pigs. Thus, in commercial production there is an increased risk of overfeeding $\mathrm{Ca}$. The effects of dietary $\mathrm{Ca}$ and $\mathrm{P}$ concentration as well as their ratio on growth performance and $P$ retention have been extensively studied in growing-finishing pigs. However, to our knowledge, such information is limited for pigs less than $15 \mathrm{~kg} \mathrm{BW}$. Therefore, the objective of this study was to evaluate the growth performance and percentage bone ash of early nursery pigs in response to different combinations of dietary STTD P and Ca concentrations provided by monocalcium $\mathrm{P}$ or phytase.

\section{MATERIALS AND METHODS}

All experimental procedures in this study were approved by the Kansas State University Institutional Animal Care and Use Committee (Manhattan, KS).

\section{Animals and Housing}

Two studies were conducted at the Cooperative Research Farm's Swine Research Nursery (Kalmbach Feeds, Inc., Sycamore, OH). Each pen $\left(1.52 \times 1.83 \mathrm{~m}^{2}\right)$ had slated metal floors and was equipped with a four-hole stainless-steel feeder and a nipple-cup waterer. Five barrows and five gilts (PIC $280 \times$ Camborough, Genus PIC, Hendersonville, TN) were housed in each pen and were allowed ad libitum access to feed and water throughout the experiments. In Exp. 1, 720 weaned pigs were used from two rooms with 36 pens per room. Upon arrival, pigs were individually weighed and assigned to pens in order to achieve balanced pen weights within room. After $4 \mathrm{~d}$ of adaptation, pens of pigs were blocked by BW (initial pig $\mathrm{BW}=6.1 \pm 0.98 \mathrm{~kg}$ ) and allotted randomly to one of six dietary treatments (Tables 2 and 3). In Exp. 2, 360 weaned pigs with initial BW of $6.0 \pm 1.08 \mathrm{~kg}$ were housed in a single room with 36 pens. Pens of pigs were allotted to one of six dietary treatments (Tables 4 and 5) in a completely randomized manner.

\section{Diets and Experimental Design}

All ingredients containing $\mathrm{Ca}$ and $\mathrm{P}$ were sampled and sent to two labs (Ward Laboratories, Inc. Kearney, NE; and Cumberland Valley Analytical Services Inc., Maugansville, MD) for analysis of $\mathrm{Ca}$ and $\mathrm{P}$ in duplicate in each lab (Table 1). The average of the four lab results for each ingredient was used in diet formulation in both experiments. In Exp. 1, the six dietary treatments were arranged in a $2 \times 3$ factorial, with two levels of $\mathrm{Ca}(0.58 \%$ and $1.03 \%)$ and three levels of STTD P $(0.33 \%$ with no phytase, 0.45 with no phytase, and $0.45 \%$ with $0.12 \%$ of the $\mathrm{P}$ assumed to be released by phytase). Diets with phytase contained 1,000 phytase units (FYT) of Ronozyme HiPhos 2500 (DSM Nutritional Products, Inc., Parsippany, NJ) with an assumed releasing value of $0.12 \%$ for $\mathrm{Ca}$ and STTD P. Pigs were fed in three phases, with the experimental diets provided in phases 1 ( $\mathrm{d} 0$ to 14$)$ and 2 (d 14 to 28 ). A common phase 3 diet was then fed to all pigs from d 28 to 42 . Nutrient and standardized ileal digestible AA digestibility coefficients used for diet formulation were obtained from NRC (2012). All diets were provided in meal form.

In Exp. 2, the six dietary treatments were arranged in a $2 \times 3$ factorial with two levels of STTD P (at or above NRC (2012) requirement estimates) and three levels of total $\mathrm{Ca}(0.65,0.90$, and $1.20 \%$ ). Pigs were fed in three phases with the experimental diets provided in phases 1 (d 0 to 10 ) and 2 (d 10 to 24), followed by a common phase 3 diet from d 24 to 45 . Diets formulated to meet NRC (2012) P requirement (NRC) contained $0.45 \%$ and $0.40 \%$ STTD P in phases 1 and 2, respectively. Diets formulated to exceed NRC (2012) P requirement 
Table 1. Analyzed $\mathrm{Ca}$ and $\mathrm{P}$ concentrations in feed ingredients (as-fed basis)

\begin{tabular}{|c|c|c|c|c|c|c|}
\hline & \multicolumn{3}{|c|}{$\mathrm{Ca}, \%$} & \multicolumn{3}{|c|}{$\mathrm{P}, \%$} \\
\hline & Lab 1* & $\mathrm{Lab} 2^{\dagger}$ & Average & Lab 1 & Lab 2 & Averag \\
\hline Corn & 0.06 & 0.02 & 0.04 & 0.28 & 0.23 & 0.26 \\
\hline Soybean meal & 0.35 & 0.31 & 0.33 & 0.63 & 0.62 & 0.62 \\
\hline HP 300 & 0.39 & 0.37 & 0.38 & 0.72 & 0.72 & 0.72 \\
\hline $\begin{array}{l}\text { Spray-dried } \\
\text { whey }\end{array}$ & 0.84 & 0.86 & 0.85 & 0.86 & 0.87 & 0.86 \\
\hline $\begin{array}{c}\text { Monocalcium } \\
\text { P }(21 \% \mathrm{P})\end{array}$ & 15.80 & 15.85 & 15.83 & 22.00 & 19.01 & 20.50 \\
\hline Limestone & 36.48 & 39.55 & 38.02 & 0.01 & 0.01 & 0.01 \\
\hline $\begin{array}{l}\text { Trace mineral } \\
\text { premix }\end{array}$ & 7.44 & 8.03 & 7.74 & 0.01 & $\mathrm{ND}^{\S}$ & 0.01 \\
\hline Vitamin premix & 12.58 & 13.69 & 13.13 & 0.07 & 0.04 & 0.05 \\
\hline Phytase $^{\#}$ & 11.77 & 12.87 & 12.32 & 0.11 & 0.05 & 0.08 \\
\hline Selenium premix & 35.66 & 40.41 & 38.04 & 0.02 & 0.02 & 0.02 \\
\hline
\end{tabular}

*Lab 1 (Ward Laboratories, Inc.); samples were analyzed in duplicates and average values were reported.

Lab 2 (Cumberland Valley Analytical Services Inc.); samples were analyzed in duplicate and average values were reported.

${ }^{\ddagger}$ Hamlet Protein, Inc., Findlay, OH.

$\$$ Not detectable.

\#Ronozyme HiPhos 2500 (DSM Nutritional Products, Inc., Parsippany, NJ).

(>NRC) contained $0.56 \%$ and $0.52 \%$ STTD P in phases 1 and 2, respectively. Diets did not contain phytase with the dietary $\mathrm{Ca}$ and $\mathrm{P}$ mainly provided by monocalcium phosphate and limestone. Phase 1 diets were prepared in pellet form and phases 2 and 3 diets were provided in meal form. Pigs and feeders were weighed at the end of each feeding phase to determine ADG, ADFI, and G:F ratio in both of the experiments.

\section{Bone Ash Analysis}

On d 21 of Exp. 1, one median-weight gilt from each pen was euthanized using a $\mathrm{CO}_{2}$ chamber and radiuses were collected. Bones were then transferred on dry ice to the Kansas State University Swine Laboratory and stored at $-20^{\circ} \mathrm{C}$ until analysis. After thawing at room temperature $\left(24^{\circ} \mathrm{C}\right)$ in plastic bags for $24 \mathrm{~h}$, bones were autoclaved for $60 \mathrm{~min}$, adhering tissue and cartilage caps were removed, then dried at $105^{\circ} \mathrm{C}$ for $7 \mathrm{~d}$. Dried radiuses were ashed in a muffle furnace at $600^{\circ} \mathrm{C}$ for $24 \mathrm{~h}$ to determine total ash weight and percentage bone ash.

\section{Chemical Analysis}

Complete diet samples were obtained and delivered to the Kansas State University Swine Laboratory, Manhattan, KS, and stored at $-20^{\circ} \mathrm{C}$ until analysis. Feed samples were analyzed for DM, $\mathrm{CP}$, ether extract, $\mathrm{Ca}$, and $\mathrm{P}$ at Ward Laboratories, Inc. Concentrations of $\mathrm{Ca}$ and $\mathrm{P}$ in complete feed samples were also analyzed at Cumberland Valley Analytical Services Inc. and Midwest Laboratories (Omaha, NE) in duplicate. Standard procedures from AOAC (2006) were followed for analysis of moisture (Method 934.01), CP (Method 990.03), ether extract (Method 920.39), Ca and P (Method 985.01). At Cumberland Valley Analytical Services Inc., AOAC (2000) method (985.01) was used for $\mathrm{Ca}$ and $\mathrm{P}$ analyses with modifications of ashing a $0.35 \mathrm{~g}$ sample for $1 \mathrm{~h}$ at $535^{\circ} \mathrm{C}$, digestion in an open crucible for $20 \mathrm{~min}$ in $15 \%$ nitric acid on a hot plate, and sample dilution to $50 \mathrm{~mL}$ and analysis on an inductively coupled plasma spectrometer (PerkinElmer 3300 XL and 5300 DV ICP; PerkinElmer Inc., Shelton, CT).

\section{Statistical Analysis}

Experiment 1 was analyzed in a randomized completely block design with a $2 \times 3$ factorial treatment structure. The statistical model contained the main effects of $\mathrm{Ca}$ and STTD P and their interactions as well as random effects of room and weight block within room. The initial statistical model included treatment and the effect of treatment within room as fixed effects. Because there was no evidence that the treatment effect was different across rooms, the treatment within room term was removed from the model and data from the two rooms were pooled in the analyses of growth performance and percentage bone ash. One pen from $0.58 \% \mathrm{Ca}+0.45 \%$ STTD $\mathrm{P}$ treatment encountered issues with feeder adjustment and had restricted feed intake as noted in the daily observation records; therefore, data from this pen were excluded from all the analyses. In Exp. 2, data were analyzed in a completely randomized design with a $2 \times 3$ factorial treatment structure. The statistical model contained the main effects of STTD P and $\mathrm{Ca}$ and their interaction. Single degree-of-freedom contrasts were performed to test the linear and quadratic effects of increasing $\mathrm{Ca}$ and their interactions with $\mathrm{P}$ concentration. All statistical analyses were conducted using the GLIMMIX procedure of SAS version 9.4 (SAS Institute, Inc., Cary, NC) with pen as the experimental unit. Means were reported as least-squares means. For response criteria with significant $\mathrm{Ca} \times$ $\mathrm{P}$ interaction, means were separated by the PDIFF option with a Tukey-Kramer adjustment. Results were considered significant at $P<0.05$ and marginally significant at $0.05<P<0.10$. 


\section{RESULTS}

\section{Chemical Analysis}

Analyzed Ca concentrations in feed ingredients were similar between the two laboratories (Table 1). However, a $15 \%$ interlaboratory discrepancy was observed for monocalcium phosphate, the primary source of $\mathrm{P}$ in the experimental diets; therefore, the average values were used in the diet formulation. It is worthy to note that significant amounts of $\mathrm{Ca}$ were included in minor ingredients, such as vitamin and trace mineral premixes, phytase, and selenium premix. However, given the small inclusion rates, these ingredients only contributed $0.03 \%$ total $\mathrm{Ca}$ in the experimental diets. The analyzed dietary $\mathrm{Ca}$ and $\mathrm{P}$ concentrations were slightly greater than the formulated values but followed similar patterns as the designed treatment structure (Tables 2-5).

\section{Experiment 1}

During phase 1 ( $\mathrm{d} 0$ to 14 ; Table 6$), \mathrm{Ca} \times \mathrm{P}$ interactions were observed for ADG and G:F $(P<0.05)$ but not for ADFI. Pigs fed diets containing $0.45 \%$ STTD $P$ with phytase had greater $(P<0.01)$ ADG than pigs fed $0.45 \%$ STTD P without phytase or pigs fed $0.33 \%$ STTD P regardless of dietary Ca concentration. The ADG of pigs fed diets containing $0.45 \%$ STTD $\mathrm{P}$ without phytase was greater $(P<0.001)$ than that of pigs fed $0.33 \%$ STTD P when diet contained high $(1.03 \%) \mathrm{Ca}$ concentration but not for diets with low $(0.58 \%) \mathrm{Ca}$ concentration. Regardless of Ca level, feeding $0.45 \%$ STTD P with phytase improved $(P<0.05)$ ADFI compared with diets with $0.33 \%$ or $0.45 \%$ STTD $\mathrm{P}$ with no phytase. Pigs fed $0.45 \%$ STTD P with or without phytase exhibited greater $(P<0.10) \mathrm{G}: F$ than pigs fed $0.33 \%$ STTD P, and the magnitude of these differences was more prominent when diets contained high $\mathrm{Ca}$ concentrations $(\mathrm{Ca} \times \mathrm{P}$ interaction, $P<0.001)$.

During phase 2 (d 14 to 28 ), $\mathrm{Ca} \times \mathrm{P}$ interactions were observed for all growth criteria $(P<0.05)$. Pigs fed diets containing $0.45 \%$ STTD P with or without phytase had greater $(P<0.05)$ ADG than those fed $0.33 \%$ STTD P when high Ca was added to diets but not for diets containing low $\mathrm{Ca}$ concentrations. When diets contained low $\mathrm{Ca}$, feeding $0.45 \%$ STTD $\mathrm{P}$ with phytase resulted in greater $(P<0.001)$ ADFI than feeding the $0.33 \%$ STTD P diet, with ADFI of pigs fed $0.45 \%$ STTD P without phytase intermediate. When fed high $\mathrm{Ca}$, ADFI of pigs fed $0.45 \%$ STTD P with or without phytase was greater
$(P<0.01)$ than those fed $0.33 \%$ STTD P. Pigs fed $0.33 \%$ STTD $P$ had lower $(P<0.001) \mathrm{G}: \mathrm{F}$ than those fed $0.45 \%$ STTD P without phytase when diets contained high Ca concentration; however, no differences were observed among low $\mathrm{Ca}$ diets.

When combining the treatment periods ( $\mathrm{d} 0$ to 28$), \mathrm{Ca} \times \mathrm{P}$ interactions were observed for all growth responses $(P<0.05)$. When low $\mathrm{Ca}$ was added to diets, feeding $0.45 \%$ STTD $P$ with phytase increased $(P<0.01)$ ADG and ADFI compared with pigs fed $0.45 \%$ STTD $\mathrm{P}$ without phytase and pigs fed $0.33 \%$ STTD P. However, with high $\mathrm{Ca}, \mathrm{ADG}$ and ADFI were similar among pigs fed $0.45 \%$ STTD P with or without phytase but were greater than those fed $0.33 \%$ STTD P diet. Gain to feed was decreased $(P<0.01)$ when low STTD P and high $\mathrm{Ca}$ were added to the diet compared with other dietary treatments. On d 28, when diets contained low Ca concentrations, pigs fed $0.45 \%$ STTD $\mathrm{P}$ with phytase had greater $(P<0.01) \mathrm{BW}$ than pigs fed $0.45 \%$ STTD P without phytase and those fed $0.33 \%$ STTD P. When diets contained high $\mathrm{Ca}, \mathrm{BW}$ was similar among pigs fed $0.45 \%$ STTD $\mathrm{P}$ with or without phytase but was greater $(P<0.01)$ than those fed $0.33 \%$ STTD P diet.

During the posttreatment period from d 28 to 42 , all pigs received a common phase 3 diet. No evidence for significant $\mathrm{Ca} \times \mathrm{P}$ interaction was observed for ADG. Pigs previously fed $1.03 \% \mathrm{Ca}$ had greater $(P<0.001)$ ADG than those previously fed $0.58 \%$ Ca. Pigs previously fed $0.33 \%$ STTD P tended to have greater $(P=0.054)$ ADG than those previously fed $0.45 \%$ STTD $\mathrm{P}$ with phytase, but similar ADG to pigs previously fed $0.45 \%$ STTD P without phytase. Pigs previously fed $0.45 \%$ STTD $\mathrm{P}$ with or without phytase had greater $(P<0.05)$ ADFI than those previously fed $0.33 \%$ STTD P, but the magnitude of these differences was greater in high $\mathrm{Ca}$ than in low $\mathrm{Ca}$ diets $(\mathrm{Ca} \times \mathrm{P}$ interaction, $P=0.063)$. For $\mathrm{G}: \mathrm{F}$, a $\mathrm{Ca} \times \mathrm{P}$ interaction $(P<0.001)$ was observed. When diets contained low Ca concentration, pigs previously fed $0.45 \%$ STTD $\mathrm{P}$ with phytase had decreased $(P=0.027) \mathrm{G}: \mathrm{F}$ compared with those previously fed $0.33 \%$ STTD P, with G:F of pigs previously fed $0.45 \%$ STTD P without phytase intermediate. When high $\mathrm{Ca}$ was added to diets, G:F was similar among pigs previously fed $0.45 \%$ STTD P with or without phytase but was poorer $(P<0.01)$ than those previously fed $0.33 \%$ STTD P.

Overall ( 0 to 42$), \mathrm{Ca} \times \mathrm{P}$ interaction was observed for all growth criteria $(P<0.10)$. Feeding $0.33 \%$ STTD P decreased $(P<0.01)$ ADG compared with feeding $0.45 \%$ STTD P with or without 
Table 2. Diet formulation, phase 1 (Exp. 1; as-fed basis)

\begin{tabular}{|c|c|c|c|c|c|c|}
\hline \multirow{2}{*}{$\frac{\text { Item }}{\mathrm{Ca}, \%:}$} & \multicolumn{6}{|c|}{ Phase 1 ( $\mathrm{d} 0$ to 14$)$} \\
\hline & 0.58 & 0.58 & $0.58^{*}$ & 1.03 & 1.03 & $1.03 *$ \\
\hline STTD P, no phytase, $\%$ : & 0.33 & 0.45 & 0.33 & 0.33 & 0.45 & 0.33 \\
\hline STTD P, with phytase, $\%$ : & - & - & 0.45 & - & - & 0.45 \\
\hline \multicolumn{7}{|l|}{ Ingredients, $\%$} \\
\hline Corn & 44.02 & 43.32 & 44.49 & 41.86 & 41.16 & 42.40 \\
\hline Soybean meal & 25.18 & 25.23 & 25.15 & 25.33 & 25.38 & 25.29 \\
\hline $\mathrm{HP} 300^{\dagger}$ & 6.00 & 6.00 & 6.00 & 6.00 & 6.00 & 6.00 \\
\hline Spray-dried whey & 20.00 & 20.00 & 20.00 & 20.00 & 20.00 & 20.00 \\
\hline Beef tallow & 2.20 & 2.45 & 2.05 & 3.00 & 3.25 & 2.80 \\
\hline Monocalcium P $(21 \% \mathrm{P})$ & 0.19 & 0.87 & 0.19 & 0.20 & 0.88 & 0.20 \\
\hline Limestone & 0.62 & 0.34 & 0.29 & 1.80 & 1.52 & 1.47 \\
\hline Salt & 0.45 & 0.45 & 0.45 & 0.45 & 0.45 & 0.45 \\
\hline L-Lys $\mathrm{HCl}$ & 0.38 & 0.38 & 0.38 & 0.38 & 0.38 & 0.38 \\
\hline DL-Met & 0.23 & 0.23 & 0.23 & 0.23 & 0.23 & 0.23 \\
\hline L-Thr & 0.18 & 0.18 & 0.18 & 0.18 & 0.18 & 0.18 \\
\hline L-Trp & 0.03 & 0.03 & 0.03 & 0.03 & 0.03 & 0.03 \\
\hline L-Val & 0.08 & 0.08 & 0.08 & 0.09 & 0.09 & 0.09 \\
\hline Trace mineral premix & 0.09 & 0.09 & 0.09 & 0.09 & 0.09 & 0.09 \\
\hline Vitamin premix ${ }^{\S}$ & 0.05 & 0.05 & 0.05 & 0.05 & 0.05 & 0.05 \\
\hline Choline chloride & 0.04 & 0.04 & 0.04 & 0.04 & 0.04 & 0.04 \\
\hline Phytase $^{\#}$ & - & - & 0.04 & - & - & 0.04 \\
\hline Zinc oxide & 0.27 & 0.27 & 0.27 & 0.27 & 0.27 & 0.27 \\
\hline Selenium & 0.02 & 0.02 & 0.02 & 0.02 & 0.02 & 0.02 \\
\hline Total & 100.00 & 100.00 & 100.00 & 100.00 & 100.00 & 100.00 \\
\hline \multicolumn{7}{|l|}{ Calculated composition } \\
\hline \multicolumn{7}{|c|}{ Standardized ileal digestible AA, \% } \\
\hline Lys & 1.40 & 1.40 & 1.40 & 1.40 & 1.40 & 1.40 \\
\hline Ile:Lys & 61 & 61 & 61 & 61 & 61 & 61 \\
\hline Leu:Lys & 116 & 116 & 116 & 115 & 115 & 115 \\
\hline Met:Lys & 37 & 37 & 37 & 37 & 37 & 37 \\
\hline Met \& Cys:Lys & 58 & 58 & 58 & 58 & 58 & 58 \\
\hline Thr:Lys & 65 & 65 & 65 & 65 & 65 & 65 \\
\hline Trp:Lys & 20 & 20 & 20 & 20 & 20 & 20 \\
\hline Val:Lys & 70 & 70 & 70 & 70 & 70 & 70 \\
\hline Total Lys, $\%$ & 1.53 & 1.53 & 1.53 & 1.53 & 1.53 & 1.53 \\
\hline $\mathrm{CP}, \%$ & 21.01 & 20.98 & 21.02 & 20.95 & 20.92 & 20.96 \\
\hline $\mathrm{NE}, \mathrm{kcal} / \mathrm{kg}$ & 2,568 & 2,568 & 2,569 & 2,569 & 2,568 & 2,568 \\
\hline $\mathrm{Ca}$, no phytase, $\%$ & 0.58 & 0.58 & 0.46 & 1.03 & 1.03 & 0.91 \\
\hline $\mathrm{Ca}$, with phytase, $\%$ & 0.58 & 0.58 & 0.58 & 1.03 & 1.03 & 1.03 \\
\hline STTD P, no phytase, $\%$ & 0.33 & 0.45 & 0.33 & 0.33 & 0.45 & 0.33 \\
\hline STTD P, with phytase, $\%$ & 0.33 & 0.45 & 0.45 & 0.33 & 0.45 & 0.45 \\
\hline Total P, $\%$ & 0.52 & 0.66 & 0.52 & 0.52 & 0.66 & 0.52 \\
\hline \multicolumn{7}{|l|}{ Analyzed composition, $\%$} \\
\hline $\mathrm{DM}$ & 91.31 & 92.17 & 91.76 & 91.52 & 91.61 & 91.22 \\
\hline $\mathrm{CP}$ & 21.90 & 20.45 & 22.15 & 21.10 & 21.55 & 21.40 \\
\hline Fat & 4.35 & 4.40 & 3.75 & 4.95 & 5.05 & 4.65 \\
\hline $\mathrm{Ca}^{\&}$ & 0.56 & 0.65 & 0.60 & 0.93 & 1.00 & 0.87 \\
\hline $\mathrm{P}^{\&}$ & 0.61 & 0.74 & 0.62 & 0.62 & 0.81 & 0.61 \\
\hline
\end{tabular}

*Phytase was added to diets at the level of 1,000 phytase units with assumed release value of $0.12 \%$ for Ca and STTD P.

${ }^{\dagger}$ Hamlet Protein, Inc., Findlay, OH.

Provided per kg of premix: $29.6 \mathrm{~g}$ Mn from manganese oxide, $104 \mathrm{~g}$ Fe from iron sulfate, $112 \mathrm{~g}$ Zn from zinc sulfate, $16 \mathrm{~g}$ Cu from copper sulfate, $1,600 \mathrm{mg}$ I from calcium iodate.

\$Provided per kg of premix: 28,659,800 IU vitamin A, 4,409,200 IU vitamin $\mathrm{D}_{3}, 105,821$ IU vitamin E, 801,665 mg vitamin K, 15,423 mg riboflavin, 66,138 mg pantothenic acid, 110,230 mg niacin, $79 \mathrm{mg}$ vitamin $\mathrm{B}_{12}, 4,409 \mathrm{mg}$ folic acid, $44 \mathrm{mg}$ thiamin, $44 \mathrm{mg}$ pyridoxine, and $4.4 \mathrm{mg}$ biotin.

\#Ronozyme HiPhos 2500 (DSM Nutritional Products, Inc., Parsippany, NJ).

\&Averaged across analyzed values from Ward Laboratories, Inc., Cumberland Valley Analytical Services Inc., and Midwest Laboratories. 
Table 3. Diet formulation, phases 2 and 3 (Exp. 1; as-fed basis)*

\begin{tabular}{|c|c|c|c|c|c|c|c|}
\hline \multirow{2}{*}{$\frac{\text { Item }}{\mathrm{Ca}, \%:}$} & \multicolumn{6}{|c|}{ Phase 2} & \multirow{2}{*}{$\frac{\text { Phase } 3}{0.86}$} \\
\hline & 0.58 & 0.58 & $0.58^{\dagger}$ & 1.03 & 1.03 & $1.03^{\dagger}$ & \\
\hline STTD P, no phytase, \%: & 0.33 & 0.45 & 0.33 & 0.33 & 0.45 & 0.33 & 0.37 \\
\hline STTD P, with phytase, $\%$ : & - & - & 0.45 & - & - & 0.45 & 0.47 \\
\hline \multicolumn{8}{|l|}{ Ingredients, $\%$} \\
\hline Corn & 52.18 & 51.48 & 52.70 & 50.04 & 49.34 & 50.56 & 59.47 \\
\hline Soybean meal & 29.54 & 29.59 & 29.50 & 29.69 & 29.74 & 29.65 & 35.15 \\
\hline HP $300^{\ddagger}$ & 3.00 & 3.00 & 3.00 & 3.00 & 3.00 & 3.00 & - \\
\hline Spray-dried whey & 10.00 & 10.00 & 10.00 & 10.00 & 10.00 & 10.00 & - \\
\hline Beef tallow & 2.20 & 2.45 & 2.00 & 3.00 & 3.25 & 2.80 & 2.00 \\
\hline Monocalcium P (21\% P) & 0.59 & 1.27 & 0.59 & 0.60 & 1.28 & 0.60 & 1.22 \\
\hline Limestone & 0.66 & 0.38 & 0.33 & 1.84 & 1.56 & 1.51 & 1.06 \\
\hline Salt & 0.50 & 0.50 & 0.50 & 0.50 & 0.50 & 0.50 & 0.35 \\
\hline L-Lys $\mathrm{HCl}$ & 0.38 & 0.38 & 0.38 & 0.38 & 0.38 & 0.38 & 0.29 \\
\hline DL-Met & 0.20 & 0.20 & 0.20 & 0.20 & 0.20 & 0.20 & 0.15 \\
\hline L-Thr & 0.18 & 0.18 & 0.18 & 0.18 & 0.18 & 0.18 & 0.13 \\
\hline L-Trp & 0.03 & 0.03 & 0.03 & 0.03 & 0.03 & 0.03 & 0.01 \\
\hline L-Val & 0.09 & 0.09 & 0.09 & 0.09 & 0.09 & 0.09 & - \\
\hline Trace mineral premix ${ }^{\S}$ & 0.09 & 0.09 & 0.09 & 0.09 & 0.09 & 0.09 & 0.09 \\
\hline Vitamin premix ${ }^{\#}$ & 0.05 & 0.05 & 0.05 & 0.05 & 0.05 & 0.05 & 0.05 \\
\hline Choline chloride & 0.04 & 0.04 & 0.04 & 0.04 & 0.04 & 0.04 & - \\
\hline Phytase $^{\&}$ & - & - & 0.04 & - & - & 0.04 & 0.02 \\
\hline Zinc oxide & 0.27 & 0.27 & 0.27 & 0.27 & 0.27 & 0.27 & - \\
\hline Selenium & 0.02 & 0.02 & 0.02 & 0.02 & 0.02 & 0.02 & 0.02 \\
\hline Total & 100.00 & 100.00 & 100.00 & 100.00 & 100.00 & 100.00 & 100.00 \\
\hline \multicolumn{8}{|l|}{ Calculated composition } \\
\hline \multicolumn{8}{|c|}{ Standardized ileal digestible AA, \% } \\
\hline Lys & 1.35 & 1.35 & 1.35 & 1.35 & 1.35 & 1.35 & 1.27 \\
\hline Ile:Lys & 61 & 61 & 61 & 61 & 60 & 61 & 64 \\
\hline Leu:Lys & 119 & 118 & 119 & 118 & 117 & 118 & 127 \\
\hline Met:Lys & 36 & 36 & 36 & 36 & 36 & 36 & 35 \\
\hline Met \& Cys:Lys & 58 & 58 & 58 & 58 & 58 & 58 & 59 \\
\hline Thr:Lys & 65 & 65 & 65 & 65 & 65 & 65 & 64 \\
\hline Trp:Lys & 20 & 20 & 20 & 20 & 20 & 20 & 20 \\
\hline Val:Lys & 71 & 71 & 71 & 71 & 71 & 71 & 68 \\
\hline Total Lys, $\%$ & 1.49 & 1.49 & 1.49 & 1.49 & 1.49 & 1.49 & 1.42 \\
\hline $\mathrm{CP}, \%$ & 20.68 & 20.66 & 20.70 & 20.61 & 20.59 & 20.63 & 20.65 \\
\hline $\mathrm{NE}, \mathrm{kcal} / \mathrm{kg}$ & 2,535 & 2,535 & 2,535 & 2,536 & 2,535 & 2,535 & 2,480 \\
\hline $\mathrm{Ca}$, no phytase, $\%$ & 0.58 & 0.58 & 0.46 & 1.03 & 1.03 & 0.91 & 0.76 \\
\hline $\mathrm{Ca}$, with phytase, $\%$ & 0.58 & 0.58 & 0.58 & 1.03 & 1.03 & 1.03 & 0.86 \\
\hline STTD P, no phytase, $\%$ & 0.33 & 0.45 & 0.33 & 0.33 & 0.45 & 0.33 & 0.37 \\
\hline STTD P, with phytase, $\%$ & 0.33 & 0.45 & 0.45 & 0.33 & 0.45 & 0.45 & 0.47 \\
\hline Total P, $\%$ & 0.54 & 0.68 & 0.55 & 0.54 & 0.68 & 0.54 & 0.62 \\
\hline \multicolumn{8}{|l|}{ Analyzed composition, $\%$} \\
\hline $\mathrm{DM}$ & 89.94 & 90.30 & 90.73 & 90.92 & 90.60 & 90.33 & 88.76 \\
\hline $\mathrm{CP}$ & 23.05 & 21.35 & 22.35 & 22.10 & 21.45 & 21.10 & 21.80 \\
\hline Fat & 4.75 & 4.50 & 4.25 & 4.80 & 4.95 & 4.45 & 4.45 \\
\hline $\mathrm{Ca}^{\mathrm{s}}$ & 0.63 & 0.65 & 0.54 & 1.32 & 1.37 & 1.13 & 0.75 \\
\hline $\mathrm{P}^{\S}$ & 0.69 & 0.78 & 0.64 & 0.65 & 0.76 & 0.62 & 0.69 \\
\hline
\end{tabular}

*Phase 2 diets were fed from d 14 to 28 and phase 3 diet were fed from d 28 to 42.

${ }^{\dagger}$ Phytase was added to diets at the level of 1,000 phytase units with assumed release value of $0.12 \%$ for Ca and STTD P.

${ }^{\sharp}$ Hamlet Protein, Inc., Findlay, OH.

\$Provided per kg of premix: $29.6 \mathrm{~g} \mathrm{Mn}$ from manganese oxide, $104 \mathrm{~g}$ Fe from iron sulfate, $112 \mathrm{~g} \mathrm{Zn}$ from zinc sulfate, $16 \mathrm{~g} \mathrm{Cu}$ from copper sulfate, $1,600 \mathrm{mg}$ I from calcium iodate.

"Provided per kg of premix: 28,659,800 IU vitamin A, 4,409,200 IU vitamin $\mathrm{D}_{3}, 105,821 \mathrm{IU}$ vitamin E, 801,665 mg vitamin K, 15,423 mg riboflavin, 66,138 mg pantothenic acid, 110,230 mg niacin, $79 \mathrm{mg}$ vitamin $\mathrm{B}_{12}, 4,409 \mathrm{mg}$ folic acid, $44 \mathrm{mg}$ thiamin, $44 \mathrm{mg}$ pyridoxine, and $4.4 \mathrm{mg}$ biotin.

\&Ronozyme HiPhos 2500 (DSM Nutritional Products, Inc., Parsippany, NJ).

${ }^{\$}$ Averaged across analyzed values from Ward Laboratories, Inc., Cumberland Valley Analytical Services Inc., and Midwest Laboratories. 
Table 4. Diet formulation, phase 1 (Exp. 2; as-fed basis)

\begin{tabular}{|c|c|c|c|c|c|c|}
\hline \multirow{3}{*}{$\begin{array}{l}\text { Item } \\
\text { STTD P: } \\
\mathrm{Ca}, \% \text { : }\end{array}$} & \multicolumn{6}{|c|}{ Phase $1(\mathrm{~d} 0$ to 10$)$} \\
\hline & \multicolumn{3}{|c|}{$\operatorname{NRC}(0.45 \%)$} & \multicolumn{3}{|c|}{$>\operatorname{NRC}(0.56 \%)$} \\
\hline & 0.65 & 0.90 & 1.20 & 0.65 & 0.90 & 1.20 \\
\hline \multicolumn{7}{|l|}{ Ingredients, $\%$} \\
\hline Corn & 46.66 & 45.54 & 44.14 & 46.04 & 44.92 & 43.53 \\
\hline Soybean meal & 21.33 & 21.40 & 21.50 & 21.38 & 21.45 & 21.55 \\
\hline HP 300* & 3.75 & 3.75 & 3.75 & 3.75 & 3.75 & 3.75 \\
\hline Fish meal & 2.50 & 2.50 & 2.50 & 2.50 & 2.50 & 2.50 \\
\hline Spray-dried whey & 20.00 & 20.00 & 20.00 & 20.00 & 20.00 & 20.00 \\
\hline Beef tallow & 2.60 & 3.00 & 3.50 & 2.80 & 3.20 & 3.70 \\
\hline Monocalcium P (21\% P) & 0.63 & 0.63 & 0.63 & 1.25 & 1.25 & 1.25 \\
\hline Limestone & 0.30 & 0.95 & 1.75 & 0.04 & 0.70 & 1.49 \\
\hline Salt & 0.50 & 0.50 & 0.50 & 0.50 & 0.50 & 0.50 \\
\hline L-Lys $\mathrm{HCl}$ & 0.48 & 0.48 & 0.48 & 0.48 & 0.48 & 0.48 \\
\hline DL-Met & 0.24 & 0.24 & 0.24 & 0.24 & 0.24 & 0.24 \\
\hline L-Thr & 0.21 & 0.21 & 0.21 & 0.21 & 0.21 & 0.21 \\
\hline L-Trp & 0.03 & 0.03 & 0.03 & 0.03 & 0.03 & 0.03 \\
\hline L-Val & 0.15 & 0.15 & 0.15 & 0.15 & 0.15 & 0.15 \\
\hline Trace mineral premix ${ }^{\dagger}$ & 0.09 & 0.09 & 0.09 & 0.09 & 0.09 & 0.09 \\
\hline Vitamin premix & 0.05 & 0.05 & 0.05 & 0.05 & 0.05 & 0.05 \\
\hline Vitamin E (20,000 IU) & 0.05 & 0.05 & 0.05 & 0.05 & 0.05 & 0.05 \\
\hline Choline chloride & 0.04 & 0.04 & 0.04 & 0.04 & 0.04 & 0.04 \\
\hline Zinc oxide & 0.39 & 0.39 & 0.39 & 0.39 & 0.39 & 0.39 \\
\hline Selenium premix & 0.02 & 0.02 & 0.02 & 0.02 & 0.02 & 0.02 \\
\hline Total & 100.00 & 100.00 & 100.00 & 100.00 & 100.00 & 100.00 \\
\hline \multicolumn{7}{|l|}{ Calculated composition } \\
\hline \multicolumn{7}{|c|}{ Standardized ileal digestible AA, \% } \\
\hline Lys & 1.40 & 1.40 & 1.40 & 1.40 & 1.40 & 1.40 \\
\hline Ile:Lys & 55 & 55 & 55 & 55 & 55 & 55 \\
\hline Leu:Lys & 109 & 108 & 108 & 109 & 108 & 107 \\
\hline Met:Lys & 38 & 38 & 38 & 38 & 38 & 38 \\
\hline Met \& Cys:Lys & 58 & 58 & 57 & 58 & 57 & 57 \\
\hline Thr:Lys & 64 & 63 & 63 & 64 & 63 & 63 \\
\hline Trp:Lys & 18 & 18 & 18 & 18 & 18 & 18 \\
\hline Val:Lys & 70 & 70 & 70 & 70 & 70 & 70 \\
\hline Total Lys, $\%$ & 1.53 & 1.53 & 1.53 & 1.53 & 1.53 & 1.53 \\
\hline $\mathrm{CP}, \%$ & 20.89 & 20.83 & 20.76 & 20.86 & 20.80 & 20.73 \\
\hline $\mathrm{NE}, \mathrm{kcal} / \mathrm{kg}$ & 2,606 & 2,606 & 2,606 & 2,606 & 2,606 & 2,606 \\
\hline $\mathrm{Ca}, \%$ & 0.65 & 0.90 & 1.20 & 0.65 & 0.90 & 1.20 \\
\hline STTD Ca ${ }^{\S}, \%$ & 0.51 & 0.67 & 0.87 & 0.53 & 0.69 & 0.88 \\
\hline $\mathrm{P}, \%$ & 0.66 & 0.65 & 0.65 & 0.78 & 0.78 & 0.77 \\
\hline STTD P, $\%$ & 0.45 & 0.45 & 0.45 & 0.56 & 0.56 & 0.56 \\
\hline Available P\#, \% & 0.42 & 0.42 & 0.42 & 0.55 & 0.55 & 0.55 \\
\hline \multicolumn{7}{|l|}{ Analyzed composition, \% } \\
\hline $\mathrm{DM}$ & 90.42 & 90.09 & 90.91 & 89.84 & 90.15 & 89.82 \\
\hline $\mathrm{CP}$ & 21.30 & 21.00 & 20.90 & 21.10 & 20.90 & 21.10 \\
\hline Fat & 5.10 & 5.30 & 6.00 & 5.10 & 5.90 & 6.00 \\
\hline $\mathrm{Ca}^{\&}$ & 0.66 & 0.80 & 1.23 & 0.66 & 0.82 & 1.27 \\
\hline $\mathrm{P}^{\&}$ & 0.64 & 0.66 & 0.66 & 0.78 & 0.80 & 0.73 \\
\hline
\end{tabular}

*Hamlet Protein, Inc., Findlay, OH.

Provided per kg of premix: 29.6 g Mn from manganese oxide, $104 \mathrm{~g}$ Fe from iron sulfate, $112 \mathrm{~g}$ Zn from zinc sulfate, $16 \mathrm{~g} \mathrm{Cu}$ from copper sulfate, $1,600 \mathrm{mg}$ I from calcium iodate.

Provided per kg of premix: 28,659,800 IU vitamin A, 4,409,200 IU vitamin $\mathrm{D}_{3}, 105,821 \mathrm{IU}$ vitamin E, 801,665 mg vitamin K, 15,423 mg riboflavin, 66,138 mg pantothenic acid, 110,230 mg niacin, $79 \mathrm{mg}$ vitamin $\mathrm{B}_{12}, 4,409 \mathrm{mg}$ folic acid, $44 \mathrm{mg}$ thiamin, $44 \mathrm{mg}$ pyridoxine, and $4.4 \mathrm{mg}$ biotin.

${ }^{\$}$ Standardized total tract digestibility coefficients for Ca content of feed ingredients were from Stein (2016).

\#Determined using availability coefficients from NRC (1998).

\&Averaged across analyzed values from Ward Laboratories, Inc., Cumberland Valley Analytical Services Inc., and Midwest Laboratories. 
Table 5. Diet formulation, phases 2 and 3 (Exp. 2; as-fed basis)*

\begin{tabular}{|c|c|c|c|c|c|c|c|}
\hline \multirow{3}{*}{$\begin{array}{l}\text { Item } \\
\text { STTD P: } \\
\text { Ca, \%: }\end{array}$} & \multicolumn{6}{|c|}{ Phase 2} & \multirow{3}{*}{$\begin{array}{r}\text { Phase } 3 \\
0.37^{\circ} \\
0.77 \\
\end{array}$} \\
\hline & \multicolumn{3}{|c|}{$\operatorname{NRC}(0.40 \%)$} & \multicolumn{3}{|c|}{$>\operatorname{NRC}(0.52 \%)$} & \\
\hline & 0.65 & 0.90 & 1.20 & 0.65 & 0.90 & 1.20 & \\
\hline \multicolumn{8}{|l|}{ Ingredients, $\%$} \\
\hline Corn & 57.76 & 56.63 & 55.23 & 57.01 & 55.87 & 54.50 & 62.60 \\
\hline Soybean meal & 24.88 & 24.96 & 25.05 & 24.93 & 25.01 & 25.10 & 32.23 \\
\hline Fish meal & 3.50 & 3.50 & 3.50 & 3.50 & 3.50 & 3.50 & - \\
\hline Spray-dried whey & 10.00 & 10.00 & 10.00 & 10.00 & 10.00 & 10.00 & - \\
\hline Beef tallow & 1.00 & 1.40 & 1.90 & 1.25 & 1.65 & 2.15 & 1.00 \\
\hline Monocalcium P $(21 \% \mathrm{P})$ & 0.61 & 0.61 & 0.61 & 1.29 & 1.29 & 1.29 & 1.25 \\
\hline Limestone & 0.38 & 1.03 & 1.83 & 0.09 & 0.75 & 1.53 & 1.10 \\
\hline Salt & 0.60 & 0.60 & 0.60 & 0.60 & 0.60 & 0.60 & 0.60 \\
\hline L-Lys $\mathrm{HCl}$ & 0.40 & 0.40 & 0.40 & 0.40 & 0.40 & 0.40 & 0.40 \\
\hline DL-Met & 0.18 & 0.18 & 0.18 & 0.18 & 0.18 & 0.18 & 0.18 \\
\hline L-Thr & 0.17 & 0.17 & 0.17 & 0.17 & 0.17 & 0.17 & 0.17 \\
\hline L-Trp & 0.03 & 0.03 & 0.03 & 0.03 & 0.03 & 0.03 & - \\
\hline L-Val & 0.10 & 0.10 & 0.10 & 0.10 & 0.10 & 0.10 & 0.07 \\
\hline Trace mineral premix ${ }^{\dagger}$ & 0.09 & 0.09 & 0.09 & 0.09 & 0.09 & 0.09 & 0.09 \\
\hline Vitamin premix & 0.05 & 0.05 & 0.05 & 0.05 & 0.05 & 0.05 & 0.05 \\
\hline Zinc oxide & 0.25 & 0.25 & 0.25 & 0.25 & 0.25 & 0.25 & 0.25 \\
\hline Selenium premix & 0.02 & 0.02 & 0.02 & 0.02 & 0.02 & 0.02 & 0.02 \\
\hline Total & 100.00 & 100.00 & 100.00 & 100.00 & 100.00 & 100.00 & 100.00 \\
\hline \multicolumn{8}{|l|}{ Calculated composition } \\
\hline \multicolumn{8}{|c|}{ Standardized ileal digestible AA, \% } \\
\hline Lys & 1.30 & 1.30 & 1.30 & 1.30 & 1.30 & 1.30 & 1.28 \\
\hline Ile:Lys & 57 & 57 & 57 & 57 & 57 & 57 & 59 \\
\hline Leu:Lys & 116 & 116 & 115 & 116 & 116 & 115 & 121 \\
\hline Met:Lys & 37 & 37 & 36 & 37 & 37 & 36 & 36 \\
\hline Met \& Cys:Lys & 58 & 57 & 57 & 57 & 57 & 57 & 58 \\
\hline Thr:Lys & 63 & 63 & 63 & 63 & 63 & 63 & 63 \\
\hline Trp:Lys & 19 & 19 & 19 & 19 & 19 & 19 & 17 \\
\hline Val:Lys & 70 & 69 & 69 & 69 & 69 & 69 & 69 \\
\hline Total Lys, $\%$ & 1.44 & 1.44 & 1.44 & 1.44 & 1.44 & 1.44 & 1.43 \\
\hline $\mathrm{CP}, \%$ & 20.70 & 20.64 & 20.58 & 20.66 & 20.61 & 20.54 & 21.19 \\
\hline $\mathrm{NE}, \mathrm{kcal} / \mathrm{kg}$ & 2,518 & 2,518 & 2,518 & 2,518 & 2,518 & 2,518 & 2,445 \\
\hline $\mathrm{Ca}, \%$ & 0.65 & 0.90 & 1.20 & 0.65 & 0.90 & 1.20 & 0.77 \\
\hline STTD Ca $\$$ \% & 0.49 & 0.65 & 0.85 & 0.51 & 0.67 & 0.86 & 0.54 \\
\hline $\mathrm{P}, \%$ & 0.62 & 0.62 & 0.62 & 0.76 & 0.75 & 0.75 & 0.61 \\
\hline STTD P, $\%$ & 0.40 & 0.40 & 0.40 & 0.52 & 0.52 & 0.52 & 0.37 \\
\hline Available $\mathrm{P}^{\#}, \%$ & 0.36 & 0.36 & 0.36 & 0.50 & 0.50 & 0.50 & 0.32 \\
\hline \multicolumn{8}{|l|}{ Analyzed composition, $\%$} \\
\hline $\mathrm{DM}$ & 89.96 & 89.24 & 89.83 & 89.61 & 90.05 & 89.35 & 88.60 \\
\hline $\mathrm{CP}$ & 21.20 & 21.10 & 21.30 & 21.10 & 21.70 & 21.20 & 21.30 \\
\hline Fat & 4.00 & 4.20 & 4.50 & 4.20 & 4.50 & 4.90 & 4.00 \\
\hline $\mathrm{Ca}^{\&}$ & 0.73 & 0.97 & 1.33 & 0.72 & 0.93 & 1.24 & 0.76 \\
\hline $\mathrm{P}^{\&}$ & 0.64 & 0.63 & 0.66 & 0.79 & 0.81 & 0.76 & 0.65 \\
\hline
\end{tabular}

*Phase 2 diets were fed from d 10 to 24 and phase 3 diet were fed from d 24 to 45 .

Provided per kg of premix: $29.6 \mathrm{~g}$ Mn from manganese oxide, $104 \mathrm{~g}$ Fe from iron sulfate, $112 \mathrm{~g}$ Zn from zinc sulfate, $16 \mathrm{~g} \mathrm{Cu}$ from copper sulfate, $1,600 \mathrm{mg}$ I from calcium iodate.

${ }^{\ddagger}$ Provided per kg of premix: 28,659,800 IU vitamin A, 4,409,200 IU vitamin $\mathrm{D}_{3}, 105,821$ IU vitamin E, 801,665 mg vitamin K, 15,423 mg riboflavin, 66,138 mg pantothenic acid, 110,230 mg niacin, $79 \mathrm{mg}$ vitamin $\mathrm{B}_{12}, 4,409 \mathrm{mg}$ folic acid, $44 \mathrm{mg}$ thiamin, $44 \mathrm{mg}$ pyridoxine, and $4.4 \mathrm{mg}$ biotin.

sTTD coefficients for Ca content of feed ingredients were from Stein (2016).

\#Determined using availability coefficients from NRC (1998).

\&Averaged across analyzed values from Ward Laboratories, Inc., Cumberland Valley Analytical Services Inc., and Midwest Laboratories. 
Table 6. Effects of $\mathrm{Ca}$ and $\mathrm{P}$ concentrations on growth performance of nursery pigs (Exp. 1)*

\begin{tabular}{|c|c|c|c|c|c|c|c|c|c|c|}
\hline \multirow{2}{*}{$\frac{\text { Item }}{\mathrm{Ca}, \%:}$} & \multicolumn{6}{|c|}{ Treatment } & \multicolumn{4}{|c|}{ Probability, $P<$} \\
\hline & 0.58 & 0.58 & $0.58^{\dagger}$ & 1.03 & 1.03 & $1.03^{\dagger}$ & & & & \\
\hline STTD P, no phytase, $\%$ : & 0.33 & 0.45 & 0.33 & 0.33 & 0.45 & 0.33 & SEM & $\mathrm{Ca} \times \mathrm{P}$ & $\mathrm{Ca}$ & $\mathrm{P}$ \\
\hline STTD P, with phytase, $\%$ : & - & - & 0.45 & - & - & 0.45 & & & & \\
\hline \multicolumn{11}{|l|}{$\mathrm{BW}, \mathrm{kg}$} \\
\hline d 0 & 6.1 & 6.1 & 6.1 & 6.1 & 6.1 & 6.1 & 0.06 & 0.773 & 0.609 & 0.208 \\
\hline d 14 & $8.9^{\mathrm{bc}}$ & $9.1^{\mathrm{b}}$ & $10.0^{\mathrm{a}}$ & $8.6^{\mathrm{c}}$ & $9.3^{\mathrm{b}}$ & $10.0^{\mathrm{a}}$ & 0.13 & 0.023 & 0.439 & 0.001 \\
\hline $\mathrm{d} 28$ & $16.4^{\mathrm{c}}$ & $16.4^{\mathrm{c}}$ & $17.8^{\mathrm{a}}$ & $14.9^{\mathrm{d}}$ & $16.8^{\mathrm{bc}}$ & $17.3^{\mathrm{ab}}$ & 0.21 & 0.001 & 0.003 & 0.001 \\
\hline $\mathrm{d} 42$ & $27.6^{\mathrm{ab}}$ & $27.6^{\mathrm{ab}}$ & $28.6^{\mathrm{a}}$ & $26.7^{\mathrm{b}}$ & $28.2^{\mathrm{a}}$ & $28.7^{\mathrm{a}}$ & 0.32 & 0.034 & 0.853 & 0.001 \\
\hline \multicolumn{11}{|l|}{ Phase 1 (d 0 to 14$)$} \\
\hline ADG, $\mathrm{g}$ & $204^{\mathrm{bc}}$ & $216^{\mathrm{b}}$ & $283^{\mathrm{a}}$ & $179^{c}$ & $231^{\mathrm{b}}$ & $279^{\mathrm{a}}$ & 7.2 & 0.019 & 0.393 & 0.001 \\
\hline ADFI, g & $272^{b}$ & $272^{\mathrm{b}}$ & $338^{\mathrm{a}}$ & $269^{b}$ & $288^{\mathrm{b}}$ & $334^{\mathrm{a}}$ & 7.2 & 0.241 & 0.594 & 0.001 \\
\hline $\mathrm{G}: \mathrm{F}, \mathrm{g} / \mathrm{kg}$ & $749^{b}$ & $794^{\mathrm{ab}}$ & $835^{\mathrm{a}}$ & $665^{\mathrm{c}}$ & $802^{\mathrm{a}}$ & $836^{\mathrm{a}}$ & 12.7 & 0.001 & 0.015 & 0.001 \\
\hline \multicolumn{11}{|l|}{ Phase 2 (d 14 to 28 ) } \\
\hline ADG, $\mathrm{g}$ & $534^{\mathrm{a}}$ & $522^{\mathrm{a}}$ & $545^{\mathrm{a}}$ & $451^{\mathrm{b}}$ & $535^{\mathrm{a}}$ & $522^{\mathrm{a}}$ & 10.5 & 0.001 & 0.001 & 0.001 \\
\hline ADFI, g & $725^{\mathrm{c}}$ & $741^{\mathrm{bc}}$ & $780^{\mathrm{ab}}$ & $712^{c}$ & $780^{\mathrm{ab}}$ & $789^{a}$ & 10.9 & 0.050 & 0.173 & 0.001 \\
\hline $\mathrm{G}: \mathrm{F}, \mathrm{g} / \mathrm{kg}$ & $737^{\mathrm{a}}$ & $704^{\mathrm{ab}}$ & $699^{\mathrm{ab}}$ & $633^{\mathrm{d}}$ & $686^{\mathrm{bc}}$ & $661^{\mathrm{cd}}$ & 8.0 & 0.001 & 0.001 & 0.165 \\
\hline \multicolumn{11}{|l|}{ Treatment (d 0 to 28 ) } \\
\hline ADG, g & $365^{\mathrm{c}}$ & $365^{\mathrm{c}}$ & $411^{\mathrm{a}}$ & $312^{\mathrm{d}}$ & $379^{\mathrm{bc}}$ & $398^{\mathrm{ab}}$ & 6.6 & 0.001 & 0.002 & 0.001 \\
\hline ADFI, g & $493^{c}$ & $501^{\mathrm{bc}}$ & $554^{\mathrm{a}}$ & $485^{c}$ & $528^{\mathrm{ab}}$ & $556^{\mathrm{a}}$ & 7.1 & 0.042 & 0.217 & 0.001 \\
\hline $\mathrm{G}: \mathrm{F}, \mathrm{g} / \mathrm{kg}$ & $740^{\mathrm{a}}$ & $729^{\mathrm{a}}$ & $742^{\mathrm{a}}$ & $642^{\mathrm{b}}$ & $718^{\mathrm{a}}$ & $715^{\mathrm{a}}$ & 6.2 & 0.001 & 0.001 & 0.001 \\
\hline \multicolumn{11}{|l|}{ Posttreatment (d 28 to 42 ) } \\
\hline $\mathrm{ADG}, \mathrm{g}$ & $800^{a b}$ & $798^{\mathrm{ab}}$ & $774^{\mathrm{b}}$ & $842^{\mathrm{a}}$ & $815^{\mathrm{ab}}$ & $816^{\mathrm{ab}}$ & 11.8 & 0.428 & 0.001 & 0.068 \\
\hline ADFI, $g$ & $1056^{c}$ & $1066^{\mathrm{ab}}$ & $1073^{\mathrm{ab}}$ & $1042^{\mathrm{c}}$ & $1121^{\mathrm{a}}$ & $1093^{\mathrm{ab}}$ & 14.7 & 0.063 & 0.093 & 0.007 \\
\hline $\mathrm{G}: \mathrm{F}, \mathrm{g} / \mathrm{kg}$ & $757^{\mathrm{b}}$ & $749^{\mathrm{bc}}$ & $722^{c}$ & $809^{a}$ & $727^{\mathrm{bc}}$ & $747^{\mathrm{bc}}$ & 9.4 & 0.001 & 0.010 & 0.001 \\
\hline \multicolumn{11}{|l|}{ d 0 to 42} \\
\hline ADG, g & $502^{\mathrm{ab}}$ & $502^{\mathrm{ab}}$ & $526^{\mathrm{a}}$ & $479^{\mathrm{b}}$ & $517^{\mathrm{a}}$ & $530^{\mathrm{a}}$ & 6.9 & 0.020 & 0.805 & 0.001 \\
\hline ADFI, g & $701^{\mathrm{c}}$ & $706^{\text {bc }}$ & $742^{\mathrm{ab}}$ & $692^{c}$ & $742^{\mathrm{ab}}$ & $750^{\mathrm{a}}$ & 9.0 & 0.044 & 0.130 & 0.001 \\
\hline $\mathrm{G}: \mathrm{F}, \mathrm{g} / \mathrm{kg}$ & $715^{\mathrm{a}}$ & $710^{\mathrm{ab}}$ & $708^{\mathrm{ab}}$ & $692^{\mathrm{b}}$ & $697^{\mathrm{b}}$ & $707^{\mathrm{ab}}$ & 4.5 & 0.055 & 0.001 & 0.616 \\
\hline Bone ash $\$ \%$ & $44.11^{\mathrm{bc}}$ & $45.62^{\mathrm{ab}}$ & $45.75^{\mathrm{ab}}$ & $42.63^{c}$ & $47.95^{\mathrm{a}}$ & $45.50^{\mathrm{ab}}$ & 0.611 & 0.007 & 0.692 & 0.001 \\
\hline
\end{tabular}

*A total of 720 mixed gender pigs (PIC $280 \times$ Camborough, Genus PIC, Hendersonville, TN) with initial BW of $6.1 \pm 0.98 \mathrm{~kg}$ were used in a 42-d growth trial with 10 pigs per pen and 12 replications (pen) per treatment. One pen from $0.58 \% \mathrm{Ca}+0.45 \%$ STTD P treatment encountered issues with feeder allowance and had restricted feed intake; therefore, data from this pen were excluded from all the analyses.

†Phytase (Ronozyme HiPhos 2500, DSM Nutritional Products, Inc., Parsippany, NJ) was added to diets at the level of 1,000 phytase units with assumed release value of $0.12 \%$ for Ca and STTD P.

${ }^{\ddagger}$ Radius samples collected from 1 median-weight gilt from each pen on $\mathrm{d} 21$.

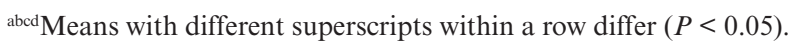

phytase, but this effect was only observed when high $\mathrm{Ca}$ was fed. For ADFI, when diets contained low Ca concentration, feeding $0.45 \%$ STTD P with phytase resulted in greater $(P=0.018)$ ADFI than feeding $0.33 \%$ STTD P diet, with that of pigs fed $0.45 \%$ STTD P without phytase intermediate. When high $\mathrm{Ca}$ was fed, ADFI of pigs fed $0.45 \%$ STTD P with or without phytase was greater $(P<0.01)$ than those fed $0.33 \%$ STTD P. Dietary STTD P level did not affect overall $\mathrm{G}: \mathrm{F}$ regardless of Ca concentration; however, G:F was decreased $(P=0.005)$ by feeding $1.03 \%$ Ca compared with feeding $0.58 \%$ $\mathrm{Ca}$ when diets contained $0.33 \%$ STTD P. This $\mathrm{Ca}$ effect was not observed when diets contained $0.45 \%$ STTD P with or without phytase. Similarly, final BW of pigs fed $0.33 \%$ STTD P was decreased
$(P<0.01)$ relative to pigs fed $0.45 \%$ STTD $P$ with or without phytase when high $\mathrm{Ca}$ was fed with no $\mathrm{P}$ response with low-dietary Ca concentration.

Pigs fed $0.33 \%$ STTD $P$ had decreased $(P<0.05)$ percentage bone ash compared with those fed $0.45 \%$ STTD P with or without phytase when high $\mathrm{Ca}$ was added to diets, but this $\mathrm{P}$ effect was not observed among treatments with low $\mathrm{Ca}$ concentration $(\mathrm{Ca} \times \mathrm{P}$ interaction, $P=0.007)$.

\section{Experiment 2}

During phase 1 ( $\mathrm{d} 0$ to 10 ), no evidence of $\mathrm{Ca} \times$ $P$ interactions were observed for any growth criteria $(P>0.38$; Table 7). Calcium and STTD P concentrations did not affect ADG or d $10 \mathrm{BW}$. However, 
Table 7. Effects of $\mathrm{Ca}$ and $\mathrm{P}$ concentrations on growth performance of nursery pigs (Exp. 2)*

\begin{tabular}{|c|c|c|c|c|c|c|c|c|c|c|c|c|}
\hline \multirow{3}{*}{$\begin{array}{l}\text { Item } \\
\text { STTD P: } \\
\text { Ca, \%: }\end{array}$} & \multicolumn{6}{|c|}{ Treatment } & \multirow{3}{*}{ SEM } & \multirow{3}{*}{$\mathrm{Ca} \times \mathrm{P}$} & \multicolumn{4}{|c|}{ Probability, $P<$} \\
\hline & \multicolumn{3}{|c|}{$\mathrm{NRC}^{\dagger}$} & \multicolumn{3}{|c|}{$>\mathrm{NRC}^{\ddagger}$} & & & \multicolumn{2}{|c|}{ Main effect } & \multicolumn{2}{|c|}{$\mathrm{Ca}$} \\
\hline & 0.65 & 0.90 & 1.20 & 0.65 & 0.90 & 1.20 & & & $\mathrm{Ca}$ & $\mathrm{P}$ & Linear & Quadratic \\
\hline \multicolumn{13}{|l|}{ BW, kg } \\
\hline d 0 & 6.0 & 6.0 & 6.0 & 6.0 & 6.0 & 6.0 & 0.01 & 0.968 & 0.989 & 0.758 & 0.897 & 0.947 \\
\hline d 10 & 6.8 & 6.8 & 6.8 & 6.8 & 6.7 & 6.7 & 0.06 & 0.833 & 0.502 & 0.129 & 0.410 & 0.406 \\
\hline d 24 & 11.5 & 11.5 & 10.7 & 11.7 & 11.5 & 11.4 & 0.19 & 0.181 & 0.018 & 0.096 & 0.006 & 0.560 \\
\hline d 45 & 25.2 & 25.5 & 24.8 & 25.3 & 25.3 & 25.2 & 0.36 & 0.644 & 0.467 & 0.756 & 0.381 & 0.388 \\
\hline \multicolumn{13}{|c|}{ Phase 1 (d 0 to 10$)$} \\
\hline ADG, $\mathrm{g}$ & 77 & 73 & 75 & 73 & 64 & 65 & 5.9 & 0.879 & 0.507 & 0.138 & 0.418 & 0.405 \\
\hline ADFI, g & 108 & 107 & 115 & 101 & 102 & 117 & 4.4 & 0.551 & 0.022 & 0.433 & 0.014 & 0.188 \\
\hline $\mathrm{G}: \mathrm{F}, \mathrm{g} / \mathrm{kg}$ & 709 & 680 & 651 & 724 & 624 & 555 & 40.3 & 0.386 & 0.029 & 0.173 & 0.009 & 0.709 \\
\hline \multicolumn{13}{|c|}{ Phase 2 (d 10 to 24 ) } \\
\hline ADG, $\mathrm{g}$ & $339^{\mathrm{a}}$ & $335^{\mathrm{a}}$ & $281^{\mathrm{b}}$ & $353^{\mathrm{a}}$ & $341^{\mathrm{a}}$ & $337^{\mathrm{a}}$ & 11.6 & $0.088^{\S}$ & 0.008 & 0.011 & 0.003 & 0.371 \\
\hline ADFI, g & 441 & 457 & 444 & 459 & 441 & 435 & 13.5 & 0.406 & 0.699 & 0.860 & 0.434 & 0.760 \\
\hline $\mathrm{G}: \mathrm{F}, \mathrm{g} / \mathrm{kg}$ & $769^{a}$ & $732^{\mathrm{a}}$ & $637^{\mathrm{b}}$ & $767^{\mathrm{a}}$ & $774^{a}$ & $773^{\mathrm{a}}$ & 16.3 & $0.001^{\#}$ & 0.002 & 0.001 & 0.001 & 0.336 \\
\hline \multicolumn{13}{|c|}{ Treatment (d 0 to 24$)$} \\
\hline ADG, $\mathrm{g}$ & 230 & 226 & 195 & 236 & 226 & 224 & 8.1 & 0.203 & 0.020 & 0.084 & 0.006 & 0.622 \\
\hline ADFI, g & 302 & 311 & 307 & 310 & 300 & 303 & 9.0 & 0.559 & 0.988 & 0.753 & 0.876 & 0.994 \\
\hline $\mathrm{G}: \mathrm{F}, \mathrm{g} / \mathrm{kg}$ & $760^{\mathrm{a}}$ & $725^{\mathrm{a}}$ & $639^{\mathrm{b}}$ & $761^{\mathrm{a}}$ & $753^{\mathrm{a}}$ & $738^{\mathrm{a}}$ & 16.3 & $0.015^{\#}$ & 0.001 & 0.003 & 0.001 & 0.439 \\
\hline \multicolumn{13}{|c|}{ Posttreatment (d 24 to 45$)$} \\
\hline ADG, g & 650 & 669 & 660 & 644 & 658 & 656 & 10.4 & 0.944 & 0.294 & 0.395 & 0.337 & 0.216 \\
\hline ADFI, g & 978 & 977 & 944 & 976 & 962 & 961 & 17.9 & 0.684 & 0.383 & 0.990 & 0.175 & 0.816 \\
\hline $\mathrm{G}: \mathrm{F}, \mathrm{g} / \mathrm{kg}$ & 665 & 685 & 699 & 660 & 684 & 685 & 9.0 & 0.747 & 0.008 & 0.354 & 0.003 & 0.269 \\
\hline \multicolumn{13}{|l|}{ d 0 to 45} \\
\hline ADG, $g$ & 426 & 431 & 411 & 425 & 427 & 425 & 8.3 & 0.532 & 0.424 & 0.622 & 0.360 & 0.349 \\
\hline ADFI, g & 618 & 619 & 603 & 618 & 609 & 610 & 11.4 & 0.758 & 0.588 & 0.939 & 0.310 & 0.908 \\
\hline $\mathrm{G}: \mathrm{F}, \mathrm{g} / \mathrm{kg}$ & 690 & 696 & 682 & 687 & 702 & 699 & 8.2 & 0.510 & 0.399 & 0.322 & 0.906 & 0.180 \\
\hline
\end{tabular}

*A total of 360 barrows and gilts (PIC $280 \times$ Camborough, Genus PIC, Hendersonville, TN) with initial BW of $6.0 \pm 1.08 \mathrm{~kg}$ were used in a 45-d trial with 10 pigs per pen and six replications (pen) per treatment.

NRC = STTD P levels formulated to meet NRC (2012) requirement estimates $(0.45 \%$ for phase 1 and $0.40 \%$ for phase 2$)$.

$\$>$ NRC $=$ STTD P levels formulated to exceed NRC (2012) requirement estimates $(0.56 \%$ for phase 1 and $0.52 \%$ for phase 2$)$.

sLinear $\mathrm{Ca} \times \mathrm{P}$ interaction: $P=0.070$; quadratic $\mathrm{Ca} \times \mathrm{P}$ interaction: $P=0.196$.

"Linear $\mathrm{Ca} \times \mathrm{P}$ interaction: $P<0.01$; quadratic $\mathrm{Ca} \times \mathrm{P}$ interaction: $P>0.10$.

${ }^{\mathrm{ab}}$ Means with different superscripts within a row differ $(P<0.05)$.

increasing Ca increased (linear, $P=0.014$ ) ADFI but decreased (linear, $P=0.009$ ) G:F.

During phase 2 (d 10 to 24 ), a marginal $\mathrm{Ca} \times$ $\mathrm{P}$ interaction was observed for $\mathrm{ADG}(P=0.088)$ and a significant interaction for G:F $(P=0.001)$, but not for ADFI or BW. Pigs fed $1.20 \% \mathrm{Ca}$ had decreased $(P<0.05)$ ADG and G:F compared with those fed $0.65 \%$ and $0.90 \% \mathrm{Ca}$ when diets contained NRC STTD P; however, this detrimental effect of high Ca was not observed in pigs fed > NRC STTD P. Average daily feed intake was not affected by dietary Ca or STTD P. Day 24 BW was decreased (linear, $P=0.006$ ) by increasing $\mathrm{Ca}$ regardless of the STTD $P$ concentration in diets. Feeding $>$ NRC STTD P resulted in a marginally greater $(P=0.096)$ d 24 BW than those fed NRC STTD P.

When combining the treatment periods $(\mathrm{d} 0$ to 24 ), no $\mathrm{Ca} \times \mathrm{P}$ interactions were observed for
ADG and ADFI. Increasing $\mathrm{Ca}$ decreased (linear, $P=0.006$ ) ADG but had no evidence for an effect on ADFI. Similarly, feeding >NRC STTD $\mathrm{P}$ marginally increased $(P=0.084)$ ADG but had no evidence for an effect on ADFI, compared with pigs fed NRC STTD P. Concentrations of $\mathrm{Ca}$ and STTD $\mathrm{P}$ had an interactive effect on G:F $(P=0.015)$. When diets contained NRC STTD $\mathrm{P}$, pigs fed $1.20 \% \mathrm{Ca}$ had poorer $(P<0.05) \mathrm{G}: \mathrm{F}$ than those fed $0.65 \%$ and $0.90 \% \mathrm{Ca}$; however, when $>$ NRC STTD P was fed, G:F was not affected by dietary $\mathrm{Ca}$ concentration.

During the posttreatment period from d 24 to 45 , all pigs received a common phase 3 diet. No interactive or main effects of $\mathrm{Ca}$ and STTD P concentrations were observed for ADG, ADFI, or final BW. However, pigs previously fed increasing dietary $\mathrm{Ca}$ had improved (linear, $P=0.003$ ) $\mathrm{G}: \mathrm{F}$ regardless 
of the STTD P content previously fed in phase 1 and 2 diets. As a result of this compensatory gain, overall (d 0 to 45) growth responses were not affected by the $\mathrm{Ca}$ and $\mathrm{P}$ concentrations fed during phases 1 and 2.

\section{DISCUSSION}

In high $\mathrm{Ca}$ diets, free $\mathrm{Ca}$ binds with $\mathrm{P}$ in the chyme to form insoluble salts, resulting in decreased digestion and absorption of dietary P (Heaney and Nordin, 2002). As an example, Stein et al. (2011) reported a linear reduction of apparent total tract digestibility of $\mathrm{P}$ from $56.9 \%$ to $46.2 \%$ when dietary $\mathrm{Ca}$ increased from $0.33 \%$ to $1.04 \%$ in growing pig. Therefore, it has been widely established that excess Ca may negatively affect pig growth performance depending on the level of $\mathrm{P}$ in diets (Reinhart and Mahan, 1986; Liu et al., 1998; González-Vega et al., 2016). The total $\mathrm{Ca}$ and STTD P requirements estimated by NRC (2012) are $0.85 \%$ and $0.45 \%$, respectively, for 5 to $7 \mathrm{~kg}$ (phase 1) pigs and $0.80 \%$ and $0.40 \%$, respectively, for 7 to $11 \mathrm{~kg}$ (phase 2) pigs. In Exp. 1, we observed that feeding 1.03\% total Ca decreased ADG, ADFI, and G:F when diets were deficient in STTD P $(0.33 \%)$, but these detrimental effects of excess $\mathrm{Ca}$ were not observed when adequate $\mathrm{P}$ diets $(0.45 \%)$ were fed. This observation is in agreement with a recent study in 100- to 130$\mathrm{kg}$ finishing pigs where excess $\mathrm{Ca}$ (total Ca:STTD $\mathrm{P}$ ratio greater than 2.2:1) in diets decreased ADG only when STTD P was at or below the NRC (2012)-estimated requirements (Merriman et al., 2017). Results from Exp. 2 suggest that increasing dietary Ca decreased G:F independent of STTD P in phase 1 . However, during phase 2, the detrimental effects of high $\mathrm{Ca}$ on ADG and G:F were only observed in pigs fed NRC STTD P $(0.40 \%)$ but not for pigs fed $0.52 \%$ STTD P. It is possible that $0.40 \%$ STTD P just met, or was marginally below, the requirement of pigs during phase 2 , which resulted in a $\mathrm{P}$ deficiency when high $\mathrm{Ca}$ was added to the diets. This marginal deficiency in STTD P is also supported by the observation that feeding high levels of STTD P (>NRC) tended to improve ADG from d 0 to 24. Vier et al. (2017) also reported that NRC (2012) may underestimate STTD P requirements for optimal performance and economic return in 11 to $25 \mathrm{~kg}$ nursery pigs.

Reinhart and Mahan (1986) observed that when diets contained low P $(0.05 \%$ below NRC), total Ca:total $\mathrm{P}$ ratios above 1.3:1 decreased growth performance of pigs in any production phase, whereas when high dietary $\mathrm{P}(0.10 \%$ above NRC) was provided, wide total Ca:total $\mathrm{P}$ ratio up to $2.0: 1$ could be fed without detrimental effects. In another study, Qian et al. (1996) observed improved growth performance of 9 to $23 \mathrm{~kg}$ pigs when total Ca:total $\mathrm{P}$ ratio was narrowed from 2.0:1 to 1.2:1 regardless of dietary $\mathrm{P}$ concentration $(0.36 \%$ or $0.45 \%$ total $\mathrm{P})$. In the present study, total Ca:total $\mathrm{P}$ ratios ranging from $0.8: 1$ to $1.6: 1$ were fed without reduction in growth performance, but decreased performance was observed when total $\mathrm{Ca}$ :total $\mathrm{P}$ ratio exceeded 1.9:1. Interestingly, during the common phases of both the experiments, pigs previously fed low STTD $\mathrm{P}$ and high $\mathrm{Ca}$ diets grew faster and were more efficient than pigs from other treatments, suggesting a compensatory gain effect in response to the increased $\mathrm{P}$ and reduced $\mathrm{Ca}$ concentrations in the phase 3 diet. However, in Exp.1, these pigs were not able to fully compensate for the negative effects of $\mathrm{P}$ deficiency when diets contained excess $\mathrm{Ca}$. In contrast to the compensatory gain observed in our study, Gonzalo et al. (2017) studied the effects of $\mathrm{P}$ depletion and repletion on growing-finishing pig performance and observed that previous $\mathrm{P}$ deficiency decreased ADG and ADFI during the subsequent repletion period.

Supplementing phytase to low $\mathrm{P}$ diets alleviated the impact of $\mathrm{P}$ deficiency on growth performance, and the magnitude of improvement was greater in diets containing high $\mathrm{Ca}$. This observation is expected because increasing STTD P above the requirement of pigs by adding phytase improves their tolerance to wide $\mathrm{Ca}: \mathrm{P}$ ratio. Moreover, the diets that included phytase to achieve $0.45 \%$ STTD $\mathrm{P}$ also improved ADG and ADFI of pigs over the diets containing $0.45 \%$ STTD P from only inorganic source, and this phytase response was more evident during phase 1 of the experiment, when dietary $\mathrm{P}$ would have been more limiting than during phase 2 . It is possible that the $0.12 \%$ release value suggested by the manufacturer for 1,000 FYT of phytase underestimated the true digestible $\mathrm{P}$ and $\mathrm{Ca}$ release, resulting in more $\mathrm{Ca}, \mathrm{P}$, or possibly other nutrients becoming available to the pig.

Dietary $\mathrm{Ca}$ concentration has also been reported to alter the releasing ability of phytase. Proposed mechanisms for a Ca-phytase interaction include: 1) formation of a Ca-phytate complex that reduces the solubility of phytate and its accessibility by phytase; 2) competition of $\mathrm{Ca}$ for active sites of the enzyme resulting in indirect repression of phytase activity; and 3) a high acid binding capacity of inorganic $\mathrm{Ca}$ sources may influence phytase activity depending on their pH activity spectrum (Selle et al., 2009). Qian et al. (1996) suggested that increasing total Ca:total $P$ ratio between 1.2:1 and 2.0:1 in diets resulted in 
approximately $1.95 \%$ reduction in the efficacy of supplemental phytase for each 0.1 unit change in $\mathrm{Ca}: \mathrm{P}$ ratio. However, the negative effects of high $\mathrm{Ca}$ on phytase activity were not observed in the present study. Feeding $1.03 \%$ total Ca to phytase-supplemented diets resulted in similar growth performance and percentage bone ash as those fed $0.58 \% \mathrm{Ca}$.

According to the $\mathrm{Ca} \times \mathrm{P}$ interaction observed for bone ash concentration, increasing dietary $\mathrm{Ca}$ exacerbated the deficiency of $\mathrm{P}$ (feeding $0.33 \%$ STTD P) for bone mineralization, compared with an improvement when diets contained adequate $\mathrm{P}(0.45 \%$ STTD P). This can be explained by the fact that a wider $\mathrm{Ca}: \mathrm{P}$ ratio (about 2.2:1) is required to form hydroxyapatite-like compounds for bone development (Crenshaw, 2001). Similar observations were reported by Létourneau-Montminy et al. (2012) where increasing dietary $\mathrm{Ca}$ from 0.5 to $0.8 \%$ decreased $\mathrm{P}$ retention by $0.016 \%$ in pigs fed a diet containing $0.1 \%$ nonphytate $\mathrm{P}$, while it increased $\mathrm{P}$ retention of pigs by $0.026 \%$ when diet contained $0.3 \%$ nonphytate P. Furthermore, the growth promoting effects of phytase were not observed for percentage bone ash. This observation is in contrast with the growth performance data, where it appeared that the $\mathrm{P}$ release by adding phytase was underestimated. Therefore, it is possible that the beneficial effect of phytase on growth performance was a result of liberating other nutrients in the diet.

In summary, our data suggest that feeding excess dietary Ca negatively affected growth performance and percentage bone ash of nursery pigs when diets are deficient in STTD P. The STTD P estimates by NRC (2012) met the requirement of nursery pigs when diets contain low $\mathrm{Ca}$ concentrations, but resulted in decreased growth performance when diets contained more than $0.90 \% \mathrm{Ca}$. Future research is in need to determine the optimal Ca:P ratio in early nursery diets. Moreover, adding inorganic $\mathrm{P}$ or phytase to $\mathrm{P}$ deficient diets improved pig performance and alleviated the negative impacts of high dietary $\mathrm{Ca}$ concentration on growth performance.

\section{LITERATURE CITED}

AOAC International. 2000. Official methods of analysis of AOAC International. 17th ed. Arlington (VA): AOAC Int.

AOAC International. 2006. Official methods of analysis of AOAC International. 18th ed. Arlington (VA): AOAC Int.

Crenshaw, T. D. 2001. Calcium, phosphorus, vitamin D, and vitamin $\mathrm{K}$ in swine nutrition. In: A. J. Lewis and L. L. Southern, editor, Swine nutrition. 2nd ed. Boca Raton (FL): CRC Press. p. 187-212.
Dersjant-Li, Y., A. Awati, H. Schulze, and G. Partridge. 2015. Phytase in non-ruminant animal nutrition: a critical review on phytase activities in the gastrointestinal tract and influencing factors. J. Sci. Food Agric. 95:878-896. doi: $10.1002 /$ jsfa. 6998

Gonçalves, M. A. D., S. S. Dritz, M. D. Tokach, J. M. DeRouchey, J. C. Woodworth, and R. D. Goodband. 2016. Fact sheets - comparing phytase sources for pigs and effects of superdosing phytase on growth performance of nursery and finishing pigs. J. Swine Health Prod. 24:97-101.

González-Vega, J. C., Y. Liu, J. C. McCann, C. L. Walk, J. J. Loor, and H. H. Stein. 2016. Requirement for digestible calcium by eleven- to twenty-five-kilogram pigs as determined by growth performance, bone ash concentration, calcium and phosphorus balances, and expression of genes involved in transport of calcium in intestinal and kidney cells. J. Anim. Sci. 94:3321-3334. doi:10.2527/ jas.2016-0444

Gonzalo, E., M. P. Létourneau-Montminy, A. Narcy, J. F. Bernier, and C. Pomar. 2017. Consequences of dietary calcium and phosphorus depletion and repletion feeding sequences on growth performance and body composition of growing pigs. Animal. 11:1-9. doi:10.1017/ S1751731117002567

Heaney, R. P., and B. E. Nordin. 2002. Calcium effects on phosphorus absorption: implications for the prevention and co-therapy of osteoporosis. J. Am. Coll. Nutr. 21:239-244.

Létourneau-Montminy, M. P., C. Jondreville, D. Sauvant, and A. Narcy. 2012. Meta-analysis of phosphorus utilization by growing pigs: effect of dietary phosphorus, calcium and exogenous phytase. Animal 6:1590-1600. doi:10.1017/ S1751731112000560

Liu, J., D. W. Bollinger, D. R. Ledoux, and T. L. Veum. 1998. Lowering the dietary calcium to total phosphorus ratio increases phosphorus utilization in low-phosphorus corn-soybean meal diets supplemented with microbial phytase for growing-finishing pigs. J. Anim. Sci. 76:808813. doi:10.2527/1998.763808x

Merriman, L. A., C. L. Walk, M. R. Murphy, C. M. Parsons, and H. H. Stein. 2017. Inclusion of excess dietary calcium in diets for 100 - to $130-\mathrm{kg}$ growing pigs reduces feed intake and daily gain if dietary phosphorus is at or below the requirement. J. Anim. Sci. 95:5439-5446. doi:10.2527/ jas2017.1995.

NRC. 1998. Nutrient Requirements of Swine. $10^{\text {th }}$ rev. ed. Washington (DC): National Academic Press.

NRC. 2012. Nutrient Requirements of Swine. $11^{\text {th }}$ rev. ed. Washington (DC): National Academic Press.

Qian, H., E. T. Kornegay, and D. E. Conner, Jr. 1996. Adverse effects of wide calcium:phosphorus ratios on supplemental phytase efficacy for weanling pigs fed two dietary phosphorus levels. J. Anim. Sci. 74:1288-1297. doi: $10.2527 / 1996.7461288 x$

Reinhart, G. A., and D. C. Mahan. 1986. Effect of various calcium:phosphorus ratios at low and high dietary phosphorus for starter, grower and finishing swine. J. Anim. Sci. 63:457-466. doi:10.2527/jas1986.632457x

Selle, P. H., A. J. Cowieson, and V. Ravindran. 2009. Consequences of calcium interactions with phytate and phytase for poultry and pigs. Livest. Sci. 124:126-141. doi:10.1016/j.livsci.2009.01.006

Stein, H. H. 2016. Calcium digestibility and requirements for digestible calcium by growing pigs. 16th Annual Midwest 
Swine Nutrition Conference Proceedings, Indianapolis, Indiana (September 9, 2016)”. p. 57-61.

Stein, H. H., O. Adeola, G. L. Cromwell, S. W. Kim, D. C. Mahan, and P. S. Miller; North Central Coordinating Committee on Swine Nutrition (NCCC-42). 2011. Concentration of dietary calcium supplied by calcium carbonate does not affect the apparent total tract digestibility of calcium, but decreases digestibility of phosphorus by growing pigs. J. Anim. Sci. 89:2139-2144. doi:10.2527/ jas. 2010-3522

Vier, C. M., F. Wu, S. S. Dritz, M. D. Tokach, M. A. D. Goncalves, U. A. D. Orlando, J. C. Woodworth, R. D. Goodband, and J. M. DeRouchey. 2017. Standardized total tract digestible phosphorus requirement of 11to 25-kg pigs. J. Anim. Sci. 95(Suppl 2):56. (Abstr.). doi:10.2527/asasmw.2017.119 